\title{
Sponsor Commercial Status
}

National Cancer Institute

\section{Source}

National Cancer Institute. Sponsor Commercial Status. NCI Thesaurus. Code C127789.

The state or condition of the sponsor as it pertains to whether the sponsor is considered a commercial entity. 